Bull. Austral. Math. Soc.

16R10, 16s36, 20M25

VOL. 57 (1998) [387-391]

\title{
SEMIGROUP RINGS IN SEMISIMPLE VARIETIES
}

\section{A.V. KelareV}

We describe semigroup rings which belong to self-dual varieties generated by a finite number of finite fields.

A variety is called semisimple if it is generated by a finite number of finite fields. Semisimple varieties play important roles in the theory of ring varieties. They occur in solutions to several natural problems and have been investigated in $[1,3,4,5,7,9,10$, $11,12,14,16,17,18]$, and other papers. On the other hand, considerable attention in the literature is devoted to semigroup algebras satisfying polynomial identities (see [8, Chapter 20]). The aim of this note is to describe semigroup algebras which belong to self-dual semisimple varieties. As a corollary, we obtain the main result of [13].

Denote by $G F\left(p^{n}\right)$ the Galois field of order $p^{n}$. The variety generated by $G F\left(p^{n}\right)$ will be denoted by $\operatorname{var}\left[G F\left(p^{n}\right)\right]$. It coincides with the variety $\operatorname{var}\left[p x=x^{p^{n}}-x=0\right]$ defined by identities $p x=x^{p^{n}}-x=0$. If $n$ divides $m$, then $G F\left(p^{m}\right) \supseteq G F\left(p^{n}\right)$.

A variety of associative rings is said to be self-dual if the lattice of its subvarieties is self-dual. It was proved in [14] that a semisimple variety is self-dual if and only if it can be generated by a finite number of finite fields with pairwise distinct characteristics. We shall consider a variety

$$
\operatorname{var}\left[G F\left(p_{1}^{n_{1}}\right), \ldots, G F\left(p_{m}^{n_{m}}\right)\right]
$$

where the primes $p_{1}, \ldots, p_{m}$ are pairwise distinct. These varieties also occur in [15].

The characteristic of a ring $R$ will be denoted by char $(R)$. A semilattice is a commutative semigroup satisfying the identity $x^{2}=x$.

TheOREM 1. Let $p_{1}, \ldots, p_{n}$ be pairwise distinct primes, $n_{1}, \ldots, n_{m}$ positive integers. A nonzero semigroup ring $R S$ belongs to the variety

$$
V=\operatorname{var}\left[G F\left(p_{1}^{n_{1}}\right), \ldots, G F\left(p_{m}^{n_{m}}\right)\right]
$$

if and only if $R \in V$ and either $S$ is a semilattice or $\operatorname{char}(R)=p_{i}$ and $S$ is a commutative semigroup satisfying the identity $x^{p_{i}^{n_{i}}}=x$.

Received 1st September, 1997

Copyright Clearance Centre, Inc. Serial-fee code: 0004-9729/98 \$A2.00+0.00. 
Note that a semigroup satisfies the identity $x^{p^{n}}=x$ if and only if it is a union of groups whose orders are divisors of $p^{n}-1$.

Let us begin with two lemmas characterising semisimple varieties. Let $Q(p)$ be the ring defined by generator $a$ and relations $p a=a^{2}=0$. The variety generated by $Q(p)$ will be denoted by $\operatorname{var}[Q(p)]$. It is the variety $\operatorname{var}[p x=x y=0]$.

Lemma 2. [4, Theorem 5] $A$ variety $V$ is generated by a finite number of finite fields if and only if it does not contain any varieties $\operatorname{var}[Q(p)]$.

If $R$ is a ring, then we put

$$
R_{p}=\left\{x \in R \mid p^{k} x=0 \text { for some } k>0\right\} .
$$

Lemma 3. Let $p_{1}, \ldots, p_{m}$ be pairwise distinct primes, $n_{1}, \ldots, n_{m}$ positive integers. Then the variety

$$
\operatorname{var}\left[G F\left(p_{1}^{n_{1}}\right), \ldots, G F\left(p_{m}^{n_{m}}\right)\right]
$$

is equal to the variety

$$
\operatorname{var}\left[p_{1} \cdots p_{m} x=0, p_{1} \cdots p_{i-1} p_{i+1} \cdots p_{m}\left(x^{p_{i}^{n_{i}}}-x\right)=0, \text { for } i=1, \ldots, m\right]
$$

and is equal to the variety

$$
\operatorname{var}\left[p_{1} \cdots p_{m} x=x \prod_{i=1}^{m}\left(x^{p_{i}^{n_{i}}-1}-1\right)=0\right] .
$$

Proof: The identities of semisimple varieties were described in [3]. For completeness we include a short self-contained proof.

Obviously, the variety (1) satisfies all identities in the definitions of (2) and (3). Therefore $(1) \subseteq(2)$ and $(1) \subseteq(3)$.

Clearly, $Q(p)$ does not satisfy all identities of the variety (2). Lemma 2 implies that the variety (2) is generated by a finite number of finite fields. Take any of these finite fields $F$. Since it satisfies the identity $p_{1} \cdots p_{m} x=0$, we get $\operatorname{char}(F)=p_{i}$ for some $1 \leqslant i \leqslant m$. The identity

$$
p_{1} \cdots p_{i-1} p_{i+1} \cdots p_{m}\left(x^{p_{i}^{n_{i}}}-x\right)=0
$$

shows that $F$ satisfies $x^{p_{i}^{n_{i}}}-x=0$. Hence $F$ belongs to $\operatorname{var}\left[G F\left(p_{i}^{n_{i}}\right)\right] \subseteq(1)$. Thus we get $(1)=(2)$.

Since $Q(p)$ does not satisfy the identities of (3), Lemma 2 tells us that the variety (3) is generated by a finite number of finite fields. Take any of these fields, say $F$. Since it satisfies the identity $p_{1} \cdots p_{m} x=0$, we get $\operatorname{char}(F)=p_{i}$ for some $1 \leqslant i \leqslant m$. Suppose that $F=G F\left(p_{i}^{n}\right)$ for a positive integer $n$. Take any element $x$ in $F$. If $x^{p_{j}{ }_{j}}-x=0$ for some $j \neq i$, then this and $x^{p_{i}^{n}}=x$ yield $x^{2}=x$; whence $x$ satisfies $x^{p_{i}^{n_{i}}}=x$. If, however, 
$x^{p_{j}^{n_{j}}}-x \neq 0$ for all $j \neq i$, then the identity $\prod_{i=1}^{m}\left(x^{p_{i}^{n_{i}}}-x\right)=0$ implies that $x^{p_{i}^{n_{i}}}=x$, again. Thus $F$ satisfies $x^{p_{i}^{n_{i}}}=x$, and so $n \leqslant n_{i}$ and $F$ belongs to $\operatorname{var}\left[G F\left(p_{i}^{n_{i}}\right)\right] \subseteq(1)$. Therefore $(1)=(3)$.

Proof of Theorem 1: Suppose that $R \in V$ and $S$ is either a semilattice or a commutative semigroup satisfying the identity $x^{p_{i}^{n_{i}}}-x=0$ if $p_{i}=\operatorname{char}(R)$. Lemma 3 shows that $\operatorname{char}(R)$ is a nonzero integer whose divisors are the $p_{i}$ that appear in the definition of $V$. In both the cases $S$ satisfies all identities $x^{p_{i}^{n_{i}}}=x$, for all $p_{i}$ dividing $\operatorname{char}(R)$. Since $R_{p}$ is a homomorphic image of $R, R \in V$ implies $R_{p} \in V$. Clearly, $R=\prod_{p \mid c h a r(R)} R_{p}$ and $R S \in V$ if and only if all the $R_{p} S \in V$, and therefore without loss of generality we may assume that $R=R_{p}$ for a prime $p=p_{1}$. Then $R$ satisfies the identity $p p_{2} \cdots p_{m} x=0$. This means the additive order of a nonzero $x \in R=R_{p}$ divides both $p p_{2} \cdots p_{m}$ and $p^{k}$ for some $k>0$, and this can only occur if its order equals $p$. Since this is true for all nonzero $x \in R$, we have $\operatorname{char}(R)=p$. A similar argument and the identity $p_{2} \cdots p_{m}\left(x^{p^{n}}-x\right)=0$, where $n=n_{1}$, of (2) in Lemma 3 imply that $R$ satisfies the identity $x^{p^{n}}-x=0$. Since $R$ has characteristic $p$, it satisfies $(x+y)^{p^{n}}=x^{p^{n}}+y^{p^{n}}$. This and the identity $x^{p^{n}}=x$ of the semigroup $S$ show that $R S$ satisfies $x^{p^{n}}=x$. Hence $R S \in V$ by Lemma 3 .

Conversely, assume that $R S \in V$. Obviously, $R \in V$, as it is a homomorphic image of $R S$. Take any $p=p_{i}$ dividing $\operatorname{char}(R)$. Then $R_{p} \neq 0$ and $R_{p} S \in V$, too. Therefore $R_{p} \in V$ and as above we see that $R_{p}$ satisfies $p x=0$. The identities in the definition of variety (2) of Lemma 3 show that $R_{p} S$ satisfies the identity $x^{p_{i}^{n_{i}}}=x$. Hence $R_{p}$ satisfies the same identity, and so it contains a nonzero idempotent $e$.

Take any $s, t \in S$. Given that $R_{p} S \in V$ and $V$ is commutative, we get $(e s)(e t)=$ (et)(es), whence $s t=t s$. Thus $S$ is commutative.

Further, take any $s$ in $S$. Substituting $e s$ for $x$ in the identity $x^{p_{i}^{n_{i}}}=x$ we see that $S$ satisfies the same identity $s_{i}^{n_{i}}=s$ as a semigroup. Thus we see that if $\operatorname{char}(R)=p_{i}$, then $S$ satisfies the identity $x^{p_{i}^{n_{i}}}-x=0$. If, however, $\operatorname{char}(R)$ is not a prime, then it has two prime divisors by Lemma 3 , say $p_{i}$ and $p_{j}$. Then $S$ satisfies the identities $x^{p_{i}^{n_{i}}}-x=x^{p_{j}^{n_{j}}}-x=0$. Therefore $S$ satisfies $x^{2}=x$, and so it is a semilattice.

The variety $\operatorname{var}\left[2 x=x^{2}-x=0\right]$ of all Boolean rings is generated by the two-element field $G F(2)$. Therefore Theorem 1 yields the following corollary.

COROLlary 4. [13] A nonzero semigroup ring $R S$ is a Boolean ring if and only if $R$ is a Boolean ring and $S$ is a semilattice.

For a prime $p$, a ring is called a $p$-ring if it satisfies the identities $p x=x^{p}-x=$ 0 (see [6, Section 33]). Since these identities define the variety generated by $G F(p)$, Theorem 1 gives us the following corollary.

COROLlaRY 5. A nonzero semigroup ring $R S$ is a p-ring if and only if $R$ is a 
$p$-ring and $S$ is a commutative semigroup satisfying $x^{p}=x$.

Following [2], we say that a semigroup $S$ stabilises a variety $V$ if $R \in V$ implies $R S \in V$. Theorem 1 immediately gives the following

COROLlaRY 6. Let $p_{1}, \ldots, p_{m}$ be pairwise distinct primes, $n_{1}, \ldots, n_{m}$ positive integers. A semigroup $S$ stabilises the variety $V=\operatorname{var}\left[G F\left(p_{1}^{n_{1}}\right), \ldots, G F\left(p_{m}^{n_{m}}\right)\right]$ if and only if either $S$ is a semilattice or $n=1$ and $S$ is a commutative semigroup satisfying the identity $x^{p_{1}^{n_{1}}}=x$.

REMARK 7. Every semigroup ring in a variety $V$ is semisimple if and only if $V$ is semisimple.

Proof: A quasi-reqular ring does not have nonzero idempotents. Therefore the variety (2) does not contain nonzero quasiregular rings. It follows that all rings in a semisimple variety are semisimple.

Conversely, suppose that a variety $V$ is not semisimple. By Lemma $2 V$ contains $\operatorname{var}[Q(p)]=\operatorname{var}[p x=x y=0]$ for a prime $p$. Take any semigroup $S$. The semigroup ring $Q(p) S$ belongs to $\operatorname{var}[Q(p)] \subseteq V$ and is not semisimple.

\section{REFERENCES}

[1] B.J. Gardner, 'Semi-simple radical classes of algebras and attainability of identities', $\mathrm{Pa}$ cific J. Math. 61 (1975), 401-416.

[2] B.J. Gardner, J.M. Osborn and I. Shestakov, 'Varieties and tensor products', Nova J. Algebra Geometry 1 (1992), 347-357.

[3] B.J. Gardner and P.N. Stewart, 'On semi-simple radical classes', Bull. Austral. Math. Soc. 13 (1975), 349-353.

[4] A.A. Iskander, 'Product of ring varieties and attainability', Trans. Amer. Math. Soc. 193 (1974), 231-238.

[5] L.M. Martynov, 'On decidable rings', Math. Notes Ural State Univ. (Sverdlovsk) 8 (1973), 82-93.

[6] N.H. McCoy, Rings and ideals, Carus Monographs Series 8 (Mathematical Society of America, 1948).

[7] G. Michler and R. Wille, 'Die primitiven Klassen arithmetischer Ringe', Math. Z. 113 (1970), 369-372.

[8] J. Okniński, Semigroup algebras (Marcel Dekker, New York, 1991).

[9] L.N. Shevrin and L.M. Martynov, 'On attainable classes of algebras', Sibirsk. Mat. Zh. 12 (1971), 1363-1381.

[10] R.L. Snider, 'Complemented hereditary radicals', Bull. Austral. Math. Soc. 4 (1971), 307-320.

[11] P.N. Stewart, 'Semi-simple radical classes', Pacific J. Math. 32 (1970), 249-254.

[12] T.R. Sundararaman, 'Lattice of precomplete varieties of rings', Proc. Kon. Nederl. Akad. Wetensch. A78 (1975), 144-148. 
[13] W.B. Vasantha, 'A note on semigroup rings which are Boolean rings', Sci. Phys. Sci. 3 (1991), 67-68.

[14] B.M. Vernikov, 'Self-dual varieties of associative rings', Ural. Gos. Univ. Mat. Zap. 14 (1985), 31-37.

[15] M.V. Volkov, 'Varieties of associative rings with the property of embeddability of amalgams', Mat. Zametki 33 (1983), 3-13.

[16] M.V. Volkov and A.V. Kelarev, 'Varieties and bands of associative algebras', Izv. Vyssh. Uchebn. Zaved. Mat. (1986), 16-23.

[17] H. Werner and R. Wille, 'Characterizierunger der primitiven Klassen arithmetischer Ringe', Math. Z. 115 (1970), 197-200.

[18] R. Wiegandt, 'Radical and semisimple classes of rings', Queen's Papers in Pure and Appl. Math. 37 (Kingston, 1974).

Department of Mathematics

University of Tasmania

GPO Box 252-37

Hobart, Tas. 7001

Australia

e-mail: kelarev@hilbert.maths.utas.edu.au 